\title{
Percutaneous endoscopic gastrostomy versus fluoroscopic gastrostomy in amyotrophic lateral sclerosis (ALS) sufferers with nutritional impairment: A meta-analysis of current studies
}

\author{
Biying Yang ${ }^{1}$ and Xiaolei Shi ${ }^{2,3}$ \\ ${ }^{1}$ Guangdong Provincial Hospital of Chinese Medicine, The Second Affiliated Hospital of Guangzhou University of Chinese \\ Medicine, Guangzhou, Guangdong, P.R. China \\ ${ }^{2}$ Department of Neurology, The First Affiliated Hospital, Yijishan Hospital of Wannan Medical College, Wuhu, Anhui, P.R. China \\ ${ }^{3}$ Kinsmen Laboratory of Neurological Research, University of British Columbia, Vancouver, BC, Canada \\ Correspondence to: Xiaolei Shi, email: shixiaolei05@gmail.com \\ Keywords: amyotrophic lateral sclerosis (ALS); gastrostomy; meta-analysis \\ Received: June 10,2017 Accepted: October 13, $2017 \quad$ Published: November 06, 2017 \\ Copyright: Yang et al. This is an open-access article distributed under the terms of the Creative Commons Attribution License 3.0 \\ (CC BY 3.0), which permits unrestricted use, distribution, and reproduction in any medium, provided the original author and source \\ are credited.
}

\section{ABSTRACT}

Gastrostomy is recommended for Amyotrophic Lateral Sclerosis (ALS) patients with malnutrition. There are two main methods of gastrostomy insertion: Percutaneous Endoscopic Gastrostomy (PEG) and Fluoroscopic Gastrostomy (FG). The latter included Radiologically Inserted Gastrostomy (RIG) and Per-oral Image-Guided Gastrostomy (PRG). A meta-analysis was conducted to compare these approaches in terms of survival outcomes, pain occurrence and success rate, through the literature search in PubMed, Web of Science and Cochrane Library. A total of 7 studies with 701 cases (322 in PEG, 264 in RIG and 115 in PRG) were enrolled in the final analysis. The lack of differences between the comparisons (PEG vs. PRG, PEG vs. RIG and PEG vs. PRG+RIG) on 30-day mortality and survival length was confirmed. For the pooling analysis of peri- and post-procedural complications, patients with PEG had a lower incidence of pain than cases with PRG and RIG together $(P<0.001)$. The same trends could be found when compared with PRG and RIG, separately $(P<0.05$ and $P<0.001$, respectively). And PEG showed a lower rate of successful attempts than PEG and RIG $(P<0.05)$. For other complications, we didn't find any differences. This meta-analysis demonstrates that PEG, PRG and RIG had their intrinsic advantages. The current evidences could not determine the preference of them. Further investigations should be done to reveal the most appropriate method for ALS patients.

\section{INTRODUCTION}

Amyotrophic Lateral Sclerosis (ALS) is the most common form of motor neuron disease in adults [1]. During the course of this disorder, malnutrition is common and act as an independent prognostic factor for survival in patients with ALS. It could be attributed to a series of progressive factors, including anorexia, dysphagia, chewing difficulty and psychological upset [2]. Approaches that maintaining a neutral nutritional balance, including altering food consistency, increasing the number of daily meals and use of feeding assistance devices, prevent malnutrition and improve physical functioning, quality of life and survival. However, as dysphagia gradually progresses, enteral feeding is often suggested for nutritional management.

Gastrostomy enteralfeeding is an effective assistance intervention approach to maintain adequate nutritional intake among these patients. The American Academy of Neurologists (AAN) and European Federation of Neurological Societies (EFNS) guidelines both recommended gastrostomy for ALS patients with nutritional deficits $[3,4]$. It is believed that gastrostomy 
feeding tube should be placed before respiratory insufficiency develops to improve survival and quality of life. There are two main methods of gastrostomy tube insertion: endoscopy and fluorescence assistedmethods, including Percutaneous Endoscopic Gastrostomy (PEG), Radiologically Inserted Gastrostomy (RIG) and Per-oral Image-Guided Gastrostomy (PRG) [5]. The two main methods are both widely used in the clinical practice for their improvement on survival status. However, studies comparing them didn't have a consistent opinion on the tube insertion method preference. Some studies believed that ALS survival status was not correlated with the method selection $[6,7]$, while some researchers found that PRG should be encouraged for a longer survival $[8,9]$. Also the complications that might occurred during and after operation, should be taken into consideration in the clinical selection. For example, PEG is often challenged for a lower insertion success rate and a higher incidence of aspiration [8]. But PEG is also to be found to have a lower rate of complications, including pain and tube replacement [10]. These controversies might hinder the application among the physicians. To solve the discrepancies, we then take this meta-analysis to demonstrate it.

\section{RESULTS}

\section{Flow of included studies}

The flow diagram was shown in Figure 1, according to the Quality of Reporting of Meta-analysesstatements with the total number of citations retrieved by the search strategy and the number included in this study. In total, 1590 articles from PubMed, Web of Science and Cochrane Library were found. After excluding the duplicates, 1144 studies were found. After further exclusion and full text assessment, 7 studies, including 701 cases (322 in PEG, 264 in RIG and 115 in PRG) fulfilling the predefined inclusion criteria, were enrolled in the final analysis (Figure 1). All publications were fulltext articles. Agreement between the two reviewers was $97 \%$ for study selection and $93 \%$ for quality assessment of trials.
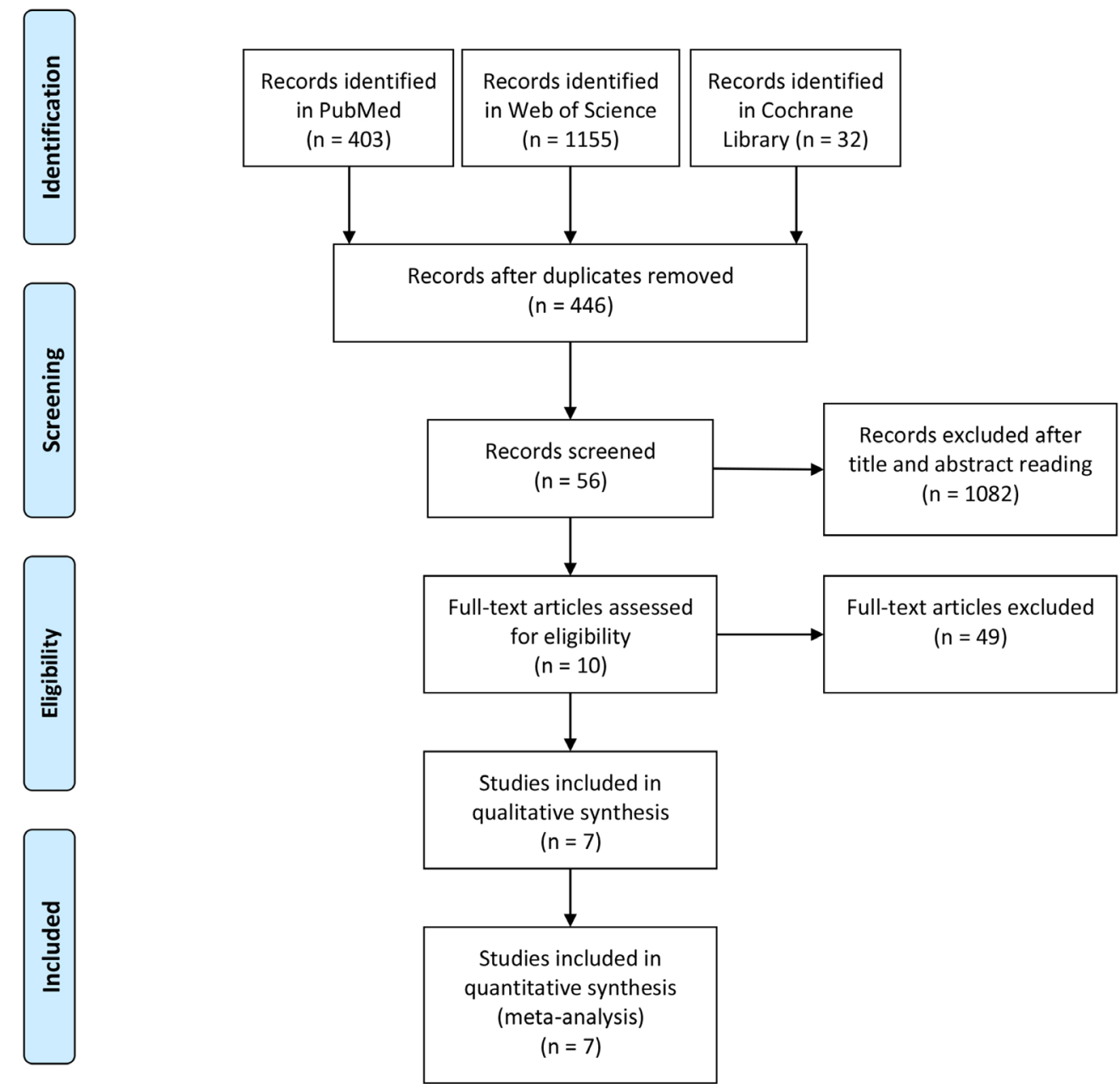

Figure 1: Flow diagram of the meta-analysis. 
Table 1: Baseline characteristics of the included studies

\begin{tabular}{|c|c|c|c|c|c|c|c|c|c|c|}
\hline Study & Year & Design & Time & Period & Geography & Group & No. & Gender (M) & $\operatorname{Age}(y)^{*}$ & Quality \\
\hline \multirow[t]{2}{*}{ Thornton [8] } & 2002 & Retro & NA & $1997.07-2001.03$ & Ireland & PEG & 11 & NA & NA & 7 \\
\hline & & & & & & PRG & 25 & NA & NA & \\
\hline \multirow[t]{2}{*}{ Chio [9] } & 2004 & Retro & $2 \mathrm{~m}$ & $2000.10-2002.12$ & Italy & PEG & 25 & 13 & $65.1 \pm 10.3$ & 7 \\
\hline & & & & & & PRG & 25 & 12 & $68.9 \pm 9.5$ & \\
\hline \multirow[t]{2}{*}{ Desport [6] } & 2005 & Pros & NA & $1999.03-2002.11$ & France & PEG & 30 & 12 & $65.7 \pm 10.3$ & 6 \\
\hline & & & & & & RIG & 20 & 4 & $66.1 \pm 9.7$ & \\
\hline \multirow[t]{2}{*}{ Shaw [12] } & 2006 & Retro & NA & 1998.11-2003.11 & UK & PEG & 18 & 9 & $62(26-85)$ & 5 \\
\hline & & & & & & RIG & 72 & 38 & $60(31-86)$ & \\
\hline \multirow[t]{2}{*}{ Blondet [7] } & 2010 & Retro & NA & 1999-2005 & France & PEG & 18 & 6 & $66.2 \pm 11.2$ & 3 \\
\hline & & & & & & PRG & 22 & 11 & $66 \pm 12$ & \\
\hline \multirow[t]{3}{*}{ Allen [10] } & 2013 & Retro & NA & $2009.01-2012.03$ & USA & PEG & 57 & 35 & $59.7 \pm 11.6$ & 8 \\
\hline & & & & & & RIG & 51 & 29 & $59.0 \pm 11.3$ & \\
\hline & & & & & & PEG & 163 & 90 & $64.2 \pm 11.7$ & 9 \\
\hline ProGas Study & & & & & & RIG & 121 & 62 & $63.6 \pm 9.8$ & \\
\hline Group [5] & 2015 & Pros & $12 \mathrm{~m}$ & $2010.11-2014.01$ & UK & PRG & 43 & 25 & $67.2 \pm 12.6$ & \\
\hline
\end{tabular}

y, year; Retro, retrospective; Pros, prospective; NA, not applicable; m, month; M, male; PEG, Percutaneous Endoscopic Gastrostomy; PRG, Per-oral ImageGuided Gastrostomy; RIG, Radiologically Inserted Gastrostomy.

\section{Study characteristics and quality assessment}

The baseline characteristics were shown in Table 1. Among all the studies included, there were 5 retrospective and 2 prospective studies. 3 studies $[8,9,11]$ compared PEG and PRG methods; 3 studies $[6,10,12]$ examined PEG and RIG procedures; and 1 study [5] assessed PEG, RIG and PRG interventions. All studies clarified the study period, and two of them indicated the follow-up time. All the cases included were from Caucasian population, with 6 studies in Europe and 1 study in North America. For quality assessment, we found that five studies scored 6 points or more, and one has a relatively large sample size.

\section{Survival analysis}

The data of 30-day mortality and survival length were shown in Table 2. 6 studies displayed 30-day mortality data after placement, and only 1 study gave 6-month mortality information. All the studies provided the survival time records after operation. But 2 of them only showed the mean or median survival time; the rest depicted as Mean $\pm \mathrm{SD}$, Median (95\% CI), Median (25th IQR). We first performed a meta-analysis of the survival data (Table 3). Compared with FG (the combination of PRG and RIG) in 6 studies, PEG had no effect on 30day mortality of the sufferers (PEG versus PRG+RIG: $5.59 \%$ versus $6.19 \%$; OR, $0.87 ; 95 \%$ CI, $0.46-1.63$; $P=0.66$ ) (Figure 2). In further analysis, we found from the pooling data of 4 studies which compared PEG and PRG, that there was no significance on 30-day mortality (PEG versus PRG: $4.15 \%$ versus $6.09 \%$; OR, $0.81 ; 95 \%$ CI, 0.28-2.30; $P=0.69$ ) (Figure 2). Also, no significant difference was found between PEG and RIG from the meta-analysis of 30-day mortality data from 3 studies (PEG versus RIG: $5.20 \%$ versus $6.25 \%$; OR, 0.83 ; $95 \%$ CI, $0.37-1.89 ; P=0.66$ ) (Figure 2). Data revealed that no marked differences of survival time were found in the comparison of patients with PEG and cases with PRG and RIG together (PEG versus PRG+RIG: WMD, 0.67; 95\% CI, $-2.63-3.97 ; P=0.69$ ) (Figure 3). Subgroup analysis also found no obvious significance in the two comparisons (PEG versus PRG: WMD, 0.34; 95\% CI, -8.12-8.81; $P=0.94$; PEG versus RIG: WMD, $1.10 ; 95 \% \mathrm{CI},-1.14$ $3.33 ; P=0.33$ ) (Figure 3).

\section{Complication analysis}

We supplied the detailed information of periand post-procedural complications in Supplementary Tables 1 and 2. The complications without clear and definite incidence information were marked as not applicable (NA). In the peri-complications (Table 3), we made the pooling analysis of difficult procedure, success attempt, $\mathrm{O}_{2}$ desaturation, distress and respiratory arrest. And we failed to assess laryngeal spasm and harmorrhage for the lack of sufficient data. The findings showed that there were no differences among all the comparisons in difficult procedure (Supplementary Figure 1), $\mathrm{O}_{2}$ desaturation (Supplementary Figure 2), distress (Supplementary Figure 3) and respiratory arrest (Supplementary Figure 4) $(P>0.05)$. However, we found that PEG operation had a lower success attempt rate than PRG and RIG together (PEG versus PRG+RIG: $88.32 \%$ versus $96.25 \%$; OR, 0.18 ; $95 \%$ CI, $0.04-0.86$; $P<0.05$ ) (Figure 5). Whereas, there were no differences 
Table 2: Clinical outcomes of all the studies

\begin{tabular}{lccc}
\hline Study & Group & 30-day mortality & Survival time \\
\hline Thornton & PEG & 1 & $11.2 \mathrm{~m}^{\mathrm{a}}$ \\
& PRG & 1 & $9.5 \mathrm{~m}^{\mathrm{a}}$ \\
Chio & PEG & 1 & $(85 \pm 12) \mathrm{d}^{\mathrm{c}}$ \\
& PRG & 1 & $(204 \pm 15) \mathrm{d}^{\mathrm{c}}$ \\
Desport & PEG & 1 & $(449 \pm 529) \mathrm{d}^{\mathrm{c}}$ \\
& RIG & 4 & $(238 \pm 178) \mathrm{d}^{\mathrm{c}}$ \\
Shaw & PEG & 2 & $7.13(4.81-9.45) \mathrm{m}^{\mathrm{d}}$ \\
& RIG & NA & $6.31(4.58-8.04) \mathrm{m}^{\mathrm{d}}$ \\
Blondet & PEG & NA & $302 \mathrm{~d}^{\mathrm{b}}$ \\
& PRG & 2 & $191 \mathrm{~d}^{\mathrm{b}}$ \\
Allen & PEG & 2 & $(10.5 \pm 7.5) \mathrm{m}^{\mathrm{c}}$ \\
& RIG & 4 & $(8.3 \pm 7.9) \mathrm{m}^{\mathrm{c}}$ \\
ProGas & PEG & 6 & $341(164) \mathrm{d}^{\mathrm{e}}$ \\
Study & RIG & 5 & $361(171) \mathrm{d}^{\mathrm{e}}$ \\
Group & PRG & 4 & $201(116) \mathrm{d}^{\mathrm{e}}$ \\
\hline
\end{tabular}

PEG, Percutaneous Endoscopic Gastrostomy; PRG, Per-oral Image-Guided Gastrostomy; RIG, Radiologically Inserted Gastrostomy; NA, Not Applicable; m, month; d, day. Survival length was depicted as Mean ${ }^{\mathrm{a}}$, Median ${ }^{\mathrm{b}}$, Mean $\pm \mathrm{SD}^{\mathrm{c}}$, Median $(95 \% \mathrm{CI})^{\mathrm{d}}$, Median $(25 \text { th IQR })^{\mathrm{e}}$; ${ }^{\mathrm{f}}$ means the incidence number in total events.

in the comparison of PEG with PRG and RIG separately (PEG versus PRG: 89.32\% versus 97.44\%; OR, 0.19; 95\% CI, 0.03-1.33; $P>0.05$; PEG versus RIG: $91.23 \%$ versus 95.83\%; OR, 0.38; 95\% CI, 0.04-3.76; $P>0.1$ ) (Figure 5).

We then focused on the post-procedural complications (Supplementary Table 2). Only infection and pain were analyzed with enough information. We found no differences in the comparison of infection occurrence $(P>0.05)$ (Supplementary Figure 5). The pooling analysis showed that patients received PEG intervention had a lower incidence of pain compared with PRG and RIG (PEG versus PRG+RIG: 20.85\% versus 41.26\%; OR, 0.42; 95\% CI, 0.27-0.67; $P<0.001)$ (Figure 4). And the same trends could be found when compared with PRG and RIG separately (PEG versus PRG: $19.34 \%$ versus $43.08 \%$; OR, 0.49 ; 95\% CI, $0.24-$ 0.98; $P<0.05$; PEG versus RIG: $31.60 \%$ versus $49.45 \%$; OR, 0.42; 95\% CI, 0.25-0.69; $P<0.001$ ) (Figure 4).

\section{Sensitivity analysis, heterogeneity and bias}

Sensitivity analysis was made in comparison with significant heterogeneity among studies (Table 3). The number of studies included in the comparisons of 30-day mortality, survival length, pain occurrence and success attempt rate were less than 10 studies. This indicated that the results were relatively unstable. The sensitivity analysis was not performed. Also, as there are less than 10 studies in allthe comparisons, a publicationbias assessment cannot be performed accurately.

\section{DISCUSSION}

To the best of our knowledge, this meta-analysis is the first to assess the role of different gastrostomy approaches on ALS patients with nutritional difficulties. Our study has some interesting and useful findings: First, there were no differences in 30-day mortality and survival length after placement between PEG and FG;Second, PEG has a higher successful placement rate with low rate of post-procedural pain occurrence. This provides basis for the recommendation of PEG in the clinical practice.

ALS, a unique neurodegenerative disease, receives more and more attention with the prevalence of ice bucket challenge in recent years. Although tremendous efforts and money have been provided, it still has no cure [13]. The nutritional deficit is widely observed among ALS population, severely impairing the living conditions of them. Gastrostomy is a commonly applied method to solve this disorder. This approachis a useful predictor for the survival status of ALS patients. Several studies had established the efficacy of these approaches. Patients with gastrostomy are likely to survive a longer time than those refusing it [14]. There are two main methods widely used in the clinical practice: PEG and FG. The latter one includes RIG and PRG [5]. Both PEG and FG 


\begin{tabular}{|c|c|c|c|c|c|c|c|}
\hline Outcome of interests & Study number & OR/WMD (95\% CI) & $P$ value & $\chi^{2}$ & df & $I^{2}, \%$ & $P$ value \\
\hline \multicolumn{8}{|l|}{ Survival } \\
\hline \multicolumn{8}{|l|}{ 30-day mortality } \\
\hline PEG vs. PRG & 4 & $0.81(0.28,2.30)$ & 0.69 & 1.49 & 3 & 0 & 0.68 \\
\hline PEG vs. RIG & 3 & $0.83(0.37,1.89)$ & 0.66 & 0.66 & 2 & 0 & 0.72 \\
\hline PEG vs. PRG+RIG & 6 & $0.87(0.46,1.63)$ & 0.66 & 1.39 & 5 & 0 & 0.93 \\
\hline \multicolumn{8}{|l|}{ Survival length } \\
\hline PEG vs. PRG & 2 & $0.34(-8.12,8.81)$ & 0.94 & 441.96 & 1 & 100 & $<0.00001$ \\
\hline PEG vs. RIG & 4 & $1.10(-1.14,3.33)$ & 0.33 & 8.51 & 3 & 65 & 0.04 \\
\hline PEG vs. PRG+RIG & 5 & $0.67(-2.63,3.97)$ & 0.69 & 144.12 & 4 & 97 & $<0.00001$ \\
\hline \multicolumn{8}{|l|}{ Peri-complication } \\
\hline \multicolumn{8}{|l|}{ Difficult procedure } \\
\hline PEG vs. PRG & 2 & $1.20(0.58,2.51)$ & 0.62 & 0.55 & 1 & 0 & 0.46 \\
\hline PEG vs. RIG & 2 & $1.59(0.81,3.16)$ & 0.18 & 0.01 & 1 & 0 & 0.91 \\
\hline PEG vs. PRG+RIG & 3 & $1.45(0.84,2.51)$ & 0.18 & 0.15 & 2 & 0 & 0.93 \\
\hline \multicolumn{8}{|l|}{ Success Attempt Rate } \\
\hline PEG vs. PRG & 4 & $0.19(0.03,1.33)$ & 0.09 & 7.18 & 3 & 58 & 0.07 \\
\hline PEG vs. RIG & 2 & $0.38(0.04,3.76)$ & 0.41 & 3.43 & 1 & 71 & 0.06 \\
\hline PEG vs. PRG+RIG & 5 & $0.18(0.04,0.86)$ & 0.03 & 10.11 & 4 & 60 & 0.04 \\
\hline \multicolumn{8}{|l|}{$\mathrm{O}_{2}$ desaturation } \\
\hline PEG vs. RIG & 2 & $2.13(0.56,8.17)$ & 0.27 & 0.02 & 1 & 0 & 0.88 \\
\hline PEG vs. PRG+RIG & 2 & $1.32(0.45,3.88)$ & 0.61 & 0.08 & 1 & 0 & 0.77 \\
\hline \multicolumn{8}{|l|}{ Distress } \\
\hline PEG vs. RIG & 2 & $1.92(0.12,30.63)$ & 0.65 & 2.83 & 1 & 65 & 0.09 \\
\hline PEG vs. PRG+RIG & 2 & $1.87(0.13,26.48)$ & 0.64 & 2.58 & 1 & 64 & 0.10 \\
\hline \multicolumn{8}{|l|}{ Respiratory arrest } \\
\hline PEG vs. PRG & 2 & $7.29(0.27,193.68)$ & 0.24 & NA & NA & NA & NA \\
\hline PEG vs. RIG & 2 & $3.77(0.41,34.92)$ & 0.24 & NA & NA & NA & NA \\
\hline PEG vs. PRG+RIG & 3 & $4.55(0.71,29.04)$ & 0.11 & 0.11 & 1 & 0 & 0.74 \\
\hline \multicolumn{8}{|l|}{ Post-complication } \\
\hline \multicolumn{8}{|l|}{ Infection } \\
\hline PEG vs. PRG & 2 & $2.20(0.67,7.19)$ & 0.19 & 0.58 & 1 & 0 & 0.45 \\
\hline PEG vs. PRG+RIG & 2 & $0.90(0.49,1.67)$ & 0.74 & 1.65 & 1 & 39 & 0.20 \\
\hline \multicolumn{8}{|l|}{ Pain } \\
\hline PEG vs. PRG & 2 & $0.49(0.24,0.98)$ & 0.04 & 0.83 & 1 & 0 & 0.36 \\
\hline PEG vs. RIG & 3 & $0.42(0.25,0.69)$ & 0.0007 & 1.69 & 2 & 0 & 0.43 \\
\hline PEG vs. PRG+RIG & 4 & $0.42(0.27,0.67)$ & 0.0002 & 2.24 & 3 & 0 & 0.52 \\
\hline
\end{tabular}

OR, odds ratio; WMD, weighted mean difference; PEG, Percutaneous Endoscopic Gastrostomy; PRG, Per-oral ImageGuided Gastrostomy; RIG, Radiologically Inserted Gastrostomy.

were widely used in the clinical practice of the disease, while whether there were any differences between them was still unknown. We further compared the improvement of survival status, and found that PEG had no difference on 30-day mortality and survival length, compared with FG or PRG and RIG, separately. They should be applied in the same place for the purpose of improving survival statues. But the limited number of studies may have 
Odds Ratio

Study or Subgroup

Thorton 2002

Chio 2004

Blondet 2010

ProGas Study Group 2015

Total $(95 \% \mathrm{CI})$

Total events

Heterogeneity: $\mathrm{Chi}^{2}=1.49, \mathrm{df}=3(\mathrm{P}=0.68) ; \mathrm{I}^{2}=0 \%$

Test for overall effect: $Z=0.40(P=0.69)$

Desport 2005

Allen 2013

ProGas Study Group 2015

$4 \quad 30$

$4 \quad 57$

5163

2
6
4

$\begin{array}{rr}20 & 16.7 \% \\ 51 & 47.4 \% \\ 121 & 35.8 \%\end{array}$

$1.38[0.23,8.38]$

$0.57[0.15,2.13]$

$0.93[0.24,3.52]$

Total $(95 \% \mathrm{CI})$

250

$192 \quad 100.0 \%$

$0.83[0.37,1.89]$

Total events 13

Test for overall effect: $Z=0.44(P=0.66)$

Thorton 2002

Chio 2004

Desport 2005

Blondet 2010

Allen 2013

ProGas Study Group 2015

Total $(95 \% \mathrm{Cl})$

Total events

Heterogeneity: $\mathrm{Chi}^{2}=1.39, \mathrm{df}=5(\mathrm{P}=0.93) ; \mathrm{I}^{2}=0 \%$

Test for overall effect: $Z=0.44(P=0.66)$

$2.40[0.14,42.26]$

$00[0.06,16.93]$

$0.42[0.10,1.84]$

$0.81[0.28,2.30]$

(2)
Odds Ratio

M-H. Fixed, $95 \% \mathrm{Cl}$

$\mathrm{Cl}$

Figure 2: Forest plot and meta-analysis of 30-day mortality.
$2.27[0.16,33.13]$

$1.00[0.07,15.12]$

$1.33[0.27,6.61]$

$1.22[0.19,7.84]$

$0.60[0.18,2.00]$

$0.72[0.23,2.22]$

\section{$307 \quad 100.0 \% \quad 0.87[0.46,1.63]$}

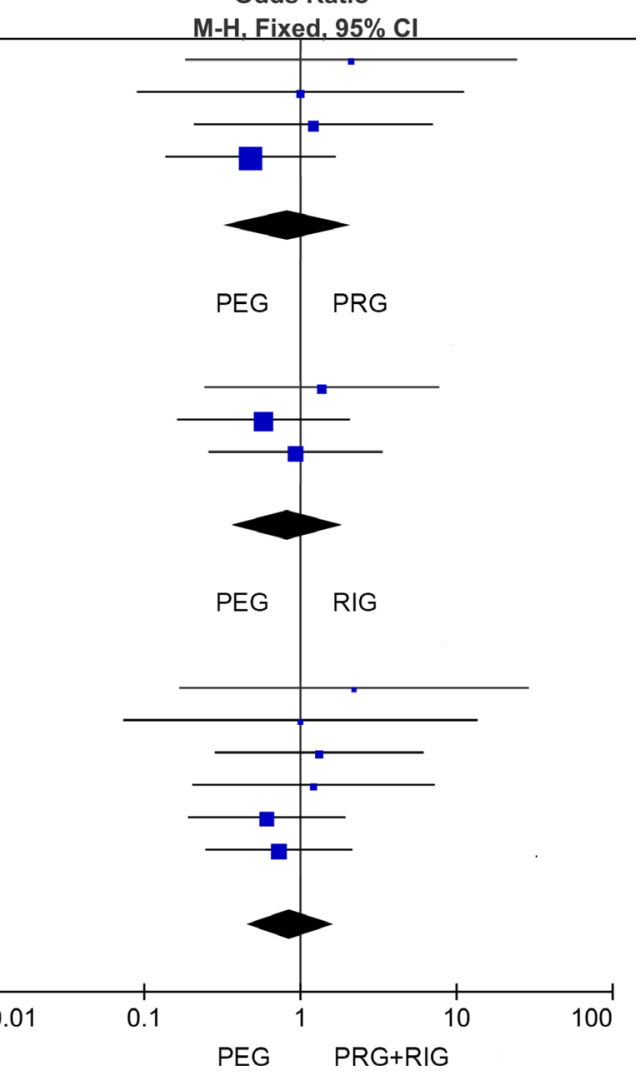




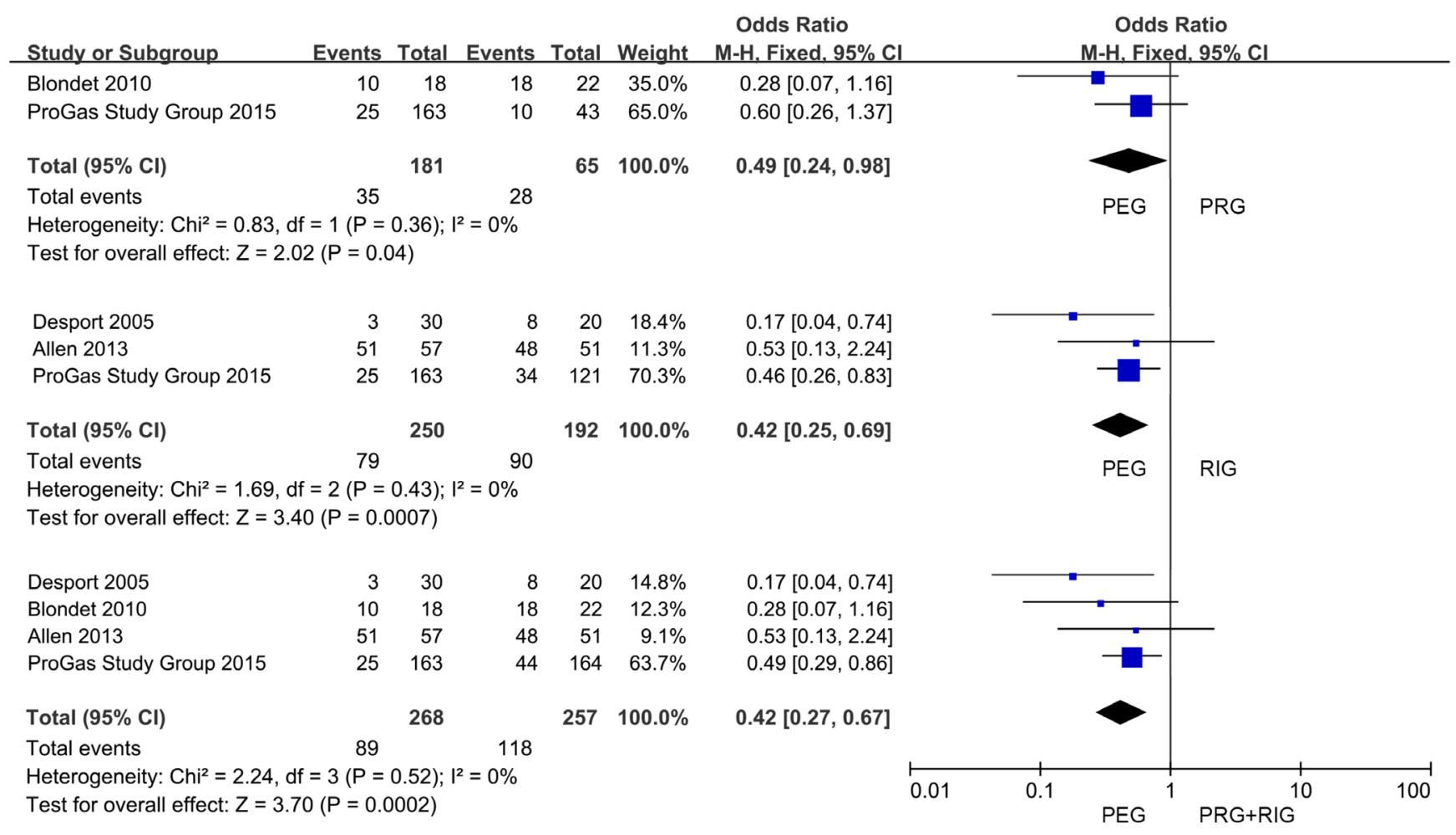

Figure 4: Forest plot and meta-analysis of pain occurrence.

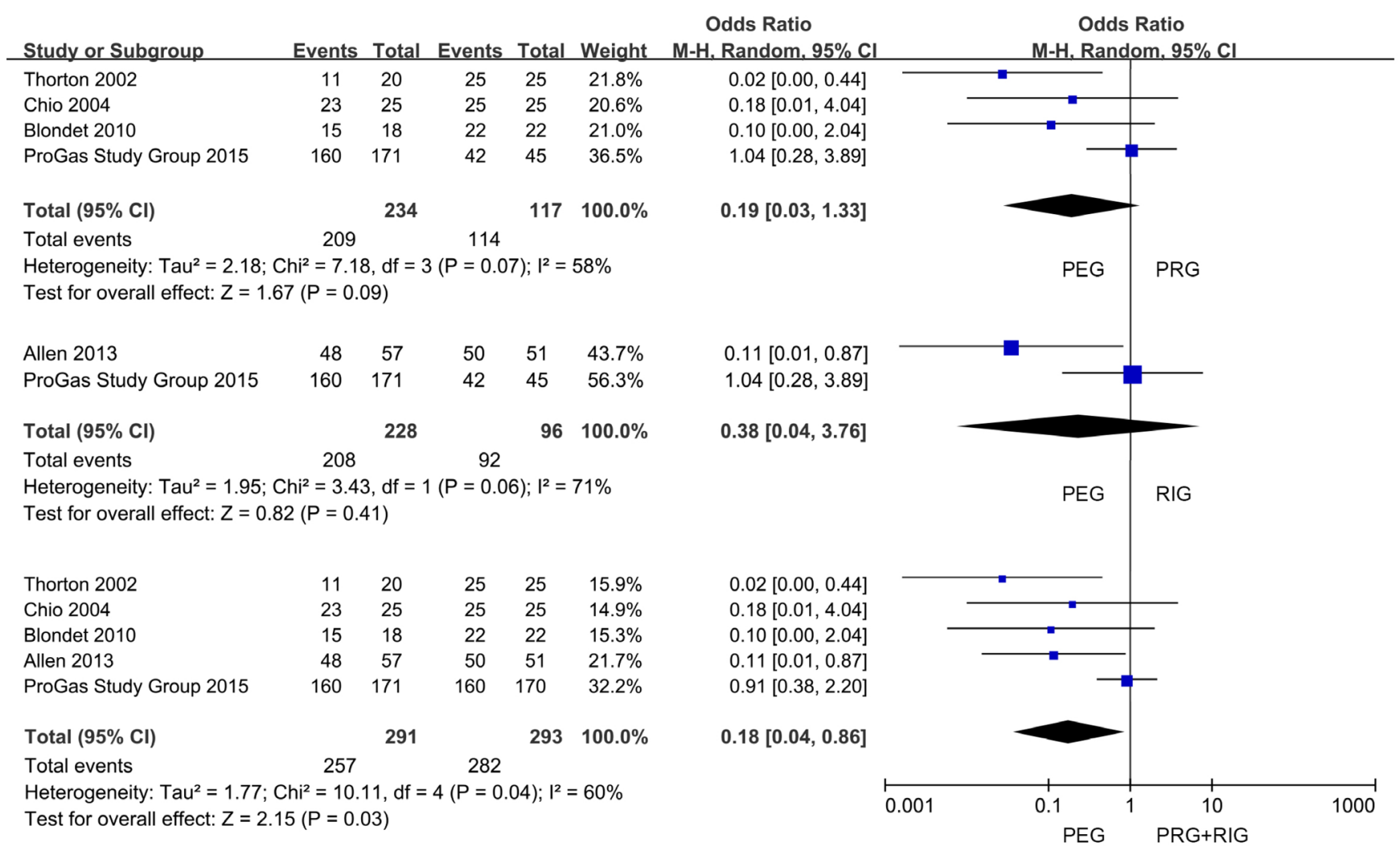

Figure 5: Forest plot and meta-analysis of success gastrostomy attempt rate. 
made observation of significant associations difficult. More studies should be found in the future for thorough analysis of the survival improvement. Although researches have indicated that gastrostomy is a safe option for the sufferers [15], it also has some concurrent incidences or complications that we would not like to meet during and after the operation. Although we would like to analyze all the peri- and post-procedural complications, the insufficient data hindered our efforts. We only examined difficult procedure, success attempt rate, $\mathrm{O}_{2}$ desaturation, distress and respiratory disorder during procedure. Many patients could not receive the gastrostomy tube successfully, because of their intrinsic anatomical structure or concomitant problems. And we found a lower successful placement rate in patients with PEG than that receiving PRG and RIG. However, no such findings were drawn when FG was assessed separately. This indicated that the fluorescent assistance facilitated and the insertion process through a precise observation of anatomical structure. Then we didn't found any significance in the pooling analysis of difficult procedure, $\mathrm{O}_{2}$ desaturation, distress and respiratory disorder. There were many complaints of post-operational complications, including infection, granulation tissue, pain, anxiety, nausea, diahhoea, pneumonia, constipation, fatigue, tube replacement, aspiration, haemorrhage, mechanical obstructive and tube migration. In our study, PEG method was found to induce a lower rate of pain incidence than FG. But due to the limited number of studies in the comparison, this finding should be more cautious to get when assessing FG, separately (PRG and RIG). It might hinder the stability of the results. And the analysis of infection revealed no differences in all the comparisons.

Most of the studies included in the study were with high quality. These pooling analyses showed significant heterogeneity and the publication bias was unable to conclude due to the limited number of studies. Subgroup analysis were undertaken to indicate the credibility of our results. However, the number of the studies included was few. This might cause an ambiguity, which required more articles to be analyzed in the future. And the background information of the patients' conditions in other systems or organs could not be got, and this should be investigated in the following clinical or meta-analysis study.

We intended to study more concurrent incidences or related complications, including economic expenses, nutritional improvements, infection incidence and so on, but the lack of raw data in these sections withdrew our attempt for a pooling analysis. We would make it in the following study to provide more interesting and meaningful findings on gastrostomy application in ALS patients.

In conclusion, PEG, PRG and RIG had their intrinsic advantages. The current evidences could not determine the preference of them. Further investigations should be done to reveal the most appropriate method for ALS patients.

\section{MATERIALS AND METHODS}

\section{Systematic literature search}

We used Preferred Reporting Items for Systematic Reviews and Meta-analysis (Supplementary Table 3) [16] and Meta-analysis of Observational Studies in Epidemiology (MOOSE) [17] recommendations for study reporting. The literature search was performed by 2 reviewers (Xiaolei Shi and Biying Yang) on articles published through to January 2017. A computerized search of the Pubmed, Web of Science, and Cochrane Library databases was performed without restriction on the language or publication type. Keywords and free text searches used the following keywords: "Percutaneous Endoscopic Gastrostomy" or "PEG" or "Fluoroscopic Gastrostomy" or "FG" or "Radiologically Inserted Gastrostomy" or "RIG" or "Per-oral Image-Guided Gastrostomy" or "PRG", in combined with "Amyotrophic Lateral Sclerosis" or "ALS". All reference sections of eligible studies and pertinent reviews were hand-reviewed for potential studies.

\section{Ethics, inclusion and exclusion criteria}

All analyses were based on previously published studies; thus, ethical approval and patient consent were not required. To insure the homogeneity across studies, included studies should met the following criteria: (1) All available prospective or retrospective comparative studies (cohort studies, case-control studies or cross-sectional studies) that included any comparison of two or three of the three methods. (2) A ALS was strictly defined according to the El Escorial criteria [18]. (3) Patients were diagnosed with nutritional decline (BMI decline, weight loss, chewing, swallowing and eating difficulties) or aspiration risk. Exclusion criteria are as following: (1) Articles without the clear definition and description of ALS diagnosis criteria and gastrostomy indications were excluded; (2) review articles, case reports, editorials, letters to editorials and experimental studies were excluded.

\section{Selection and data extraction}

Data from the included studies were extracted and summarized independently by the two authors (Xiaolei Shi and Biying Yang). Any disagreement was resolved by consensus-based discussion. Data including survival information (30-day mortality and survival time), peri(difficult procedure, success attempt, $\mathrm{O}_{2}$ desaturation, distress, respiratory arrest, laryngeal spasm and haemorrhage) and post-procedural (infection, granulation 
tissue, pain, anxiety, nausea, diahhoea, pneumonia, constipation, fatigue, replacement, aspiration, haemorrhage and mechanical obstruction/tube migration) complications. The primary outcomes were the comparison of the data (30-day mortality, survival time, successful attempt rate, infection and pain) between PEG and FG (PRG and RIG). 30-day mortalityis defined as the mortality rate of patients 30 days after the placement of gastrostomy tube. Survival time is the living duration time since the operation procedure. Successful attempt rate means the success rate of tube insertion among patients with PEG and FG. Pain and infection occurrence means the incidence of them after the placement.

\section{Quality assessment and statistical analyses}

The quality of observationalstudies was assessed by the Newcastle-Ottawa Quality Assessment Scale [19], which consists of three factors: Patient selection, comparability of the study groups, and assessment of outcome. A score of 0-9 was allocated to each study. Studies that achieved six or more stars were considered to be of high quality. The quality of cross-sectional studies was assessed by an 11-item checklist which was recommended by Agency for Healthcare Research and Quality (AHRQ) [20]. An item would be scored " 0 " if it was answered "NO" or "UNCLEAR"; if it was answered "YES", then the item scored " 1 ". Article quality was assessed as follows: low quality $=0-3$; moderate quality $=$ $4-7$; high quality $=8-11$. Meta-analysis was performed on studies that provided data on outcomes of patients using the software package Revman 5. The subgroup analysis were undertaken in the comparison of these factors in PEG with PRG or RIG. The weighted mean difference (WMD) and odds ratio (OR) were used to compare continuous and dichotomous variables, respectively. All results were reported with $95 \%$ confidence intervals (CIs). Statistical heterogeneity was assessed using the $I^{2}$ statistic with a Cochran $Q$-test, which describes the proportion of total variation that is attributable to differences among trials rather than sampling error (chance). An $I^{2}$ value of $<25 \%$ was defined to represent low heterogeneity, a value between $25 \%$ and $50 \%$ was defined as moderate heterogeneity and $>50 \%$ was defined as high heterogeneity. The random-effects model was used if there was high heterogeneity between studies. Otherwise, the fixed-effects model was used. As there were less than 10 studies in this meta-analysis, we didn't do sensitivity analysis and publication bias.

\section{Author contributions}

XLS and BYY conceived the idea and designed the study. They did the article search, extracted and then checked the data. And the statistical analysis was done by the two. XLS wrote the manuscript. All authors read and approved the final submission of the manuscript.

\section{CONFLICTS OF INTEREST}

There are no conflicts among the authors.

\section{FUNDING}

This study was supported by grants from the Scientific Research Program of Traditional Chinese Medicine Bureau of Guangdong Province (Grant No. 20161080).

\section{REFERENCES}

1. Williams DB, Windebank AJ. Motor neuron disease (amyotrophic lateral sclerosis). Mayo Clin Proc. 1991; 66:54-82.

2. Marin B, Desport JC, Kajeu P, Jesus P, Nicolaud B, Nicol M, Preux PM, Couratier P. Alteration of nutritional status at diagnosis is a prognostic factor for survival of amyotrophic lateral sclerosis patients. J Neurol Neurosurg Psychiatry. 2011; 82:628-634.

3. Bede P, Oliver D, Stodart J, van den Berg L, Simmons Z, O Brannagáin D, Borasio GD, Hardiman O. Palliative care in amyotrophic lateral sclerosis: a review of current international guidelines and initiatives. BMJ Support Palliat Care. 2011; 1:343-348.

4. Miller RG, Jackson CE, Kasarskis EJ, England JD, Forshew D, Johnston W, Kalra S, Katz JS, Mitsumoto H, Rosenfeld J, Shoesmith C, Strong MJ, Woolley SC. Practice parameter update: the care of the patient with amyotrophic lateral sclerosis: drug, nutritional, and respiratory therapies (an evidence-based review): report of the Quality Standards Subcommittee of the American Academy of Neurology. Neurology. 2009; 73:1218-1226.

5. ProGas Study Group. Gastrostomy in patients with amyotrophic lateral sclerosis (ProGas): a prospective cohort study. Lancet Neurol. 2015; 14:702-709.

6. Desport JC, Mabrouk T, Bouillet P, Perna A, Preux PM, Couratier P. Complications and survival following radiologically and endoscopically-guided gastrostomy in patients with amyotrophic lateral sclerosis. Amyotroph Lateral Scler Other Motor Neuron Disord. 2005; 6:88-93.

7. Blondet A, Lebigot J, Nicolas G, Boursier J, Person B, Laccoureye L, Aube C. Radiologic versus endoscopic placement of percutaneous gastrostomy in amyotrophic lateral sclerosis: multivariate analysis of tolerance, efficacy, and survival. J Vasc Interv Radiol. 2010; 21:527-533.

8. Thornton FJ, Fotheringham T, Alexander M, Hardiman O, McGrath FP, Lee MJ. Amyotrophic lateral sclerosis: enteral nutrition provision - endoscopic or radiologic gastrostomy? Radiology. 2002; 224:713-717. 
9. Chio A, Galletti R, Finocchiaro C, Righi D, Ruffino MA, Calvo A, Di Vito N, Ghiglione P, Terreni AA, Mutani R. Percutaneous radiological gastrostomy: a safe and effective method of nutritional tube placement in advanced ALS. J Neurol Neurosurg Psychiatry. 2004; 75:645-647.

10. Allen JA, Chen R, Ajroud-Driss S, Sufit RL, Heller S, Siddique T, Wolfe L. Gastrostomy tube placement by endoscopy versus radiologic methods in patients with ALS: a retrospective study of complications and outcome. Amyotroph Lateral Scler Frontotemporal Degener. 2013; 14:308-314.

11. Chavada G, El-Nayal A, Lee F, Webber SJ, McAlindon M, Walsh T, Hollinger H, McDermott CJ, Shaw PJ. Evaluation of two different methods for per-oral gastrostomy tube placement in patients with motor neuron disease (MND): PIG versus PEG procedures. Amyotroph Lateral Scler. 2010; 11:531-536.

12. Shaw AS, Ampong MA, Rio A, Al-Chalabi A, Sellars ME, Ellis C, Shaw CE, Leigh NP, Sidhu PS. Survival of patients with ALS following institution of enteral feeding is related to pre-procedure oximetry: a retrospective review of 98 patients in a single centre. Amyotroph Lateral Scler. 2006; 7:16-21.

13. Vaidya M. Ice bucket challenge cash may help derisk ALS drug research. Nat Med. 2014; 20:1080.

14. Knibb JA, Keren N, Kulka A, Leigh PN, Martin S, Shaw CE, Tsuda M, Al-Chalabi A. A clinical tool for predicting survival in ALS. J Neurol Neurosurg Psychiatry. 2016; 87:1361-1367.
15. Blondet A, Lebigot J, Nicolas G, Boursier J, Person B, Laccoureye L, Aube C. Radiologic versus endoscopic placement of percutaneous gastrostomy in amyotrophic lateral sclerosis: multivariate analysis of tolerance, efficacy, and survival. J Vasc Interv Radiol. 2010; 21:527-533.

16. Panic N, Leoncini E, de Belvis G, Ricciardi W, Boccia S. Evaluation of the endorsement of the preferred reporting items for systematic reviews and meta-analysis (PRISMA) statement on the quality of published systematic review and meta-analyses. PLoS One. 2013; 8:e83138.

17. Stroup DF, Berlin JA, Morton SC, Olkin I, Williamson GD, Rennie D, Moher D, Becker BJ, Sipe TA, Thacker SB. Meta-analysis of observational studies in epidemiology: a proposal for reporting. Meta-analysis Of Observational Studies in Epidemiology (MOOSE) group. JAMA. 2000; 283:2008-2012.

18. Agosta F, Al-Chalabi A, Filippi M, Hardiman O, Kaji R, Meininger V, Nakano I, Shaw P, Shefner J, van den Berg LH, Ludolph A. The El Escorial criteria: strengths and weaknesses. Amyotroph Lateral Scler Frontotemporal Degener. 2015; 16:1-7.

19. Wells G, Shea BJ, O'Connell D, Peterson J, Welch V, Losos M, Tugwell P. The Newcastle-Ottawa Scale (NOS) for assessing the quality of nonrandomised studies in metaanalyses. AEA. 2014; 18:727-734.

20. Hartling L, Ospina M, Liang Y, Dryden DM, Hooton N, Krebs SJ, Klassen TP. Risk of bias versus quality assessment of randomised controlled trials: cross sectional study. BMJ. 2009; 339:b4012. 